\title{
Research Paper \\ The Effects of Holographic Reprocessing Therapy on Cognitive Flexibility and Posttraumatic Growth in Women With Breast Cancer
}

\author{
Seifollah Aghajani ${ }^{1}$ (1), *Sahar Khoshsorour ${ }^{1}$ (i) Sara Taghizadeh Hir ${ }^{1}$ (i) \\ 1. Department of Psychology, Faculty of Educational Sciences and Psychology, University of Mohaghegh Ardabili, Ardabil, Iran.
}

\begin{tabular}{|c|c|}
\hline $\begin{array}{l}\text { Use your device to scan } \\
\text { and read the article online }\end{array}$ & Cltation: Aghajani S, Khoshsorour S, Taghizadeh Hir S. [The Effects of Holographic Reprocessing Therapy on Cognitive \\
\hline 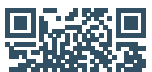 & $\begin{array}{l}\text { Flexibility and Posttraumatic Growth in Women With Breast Cancer (Persian)]. Journal of Arak University of Medical Sciences } \\
\text { (JAMS). 2021; 24(1):108-121. https://doi.org/10.32598/JAMS.24.1.5880.2 }\end{array}$ \\
\hline arifing & doi'https://doi.org/10.32598/JAMS.24.1.5880.2 \\
\hline
\end{tabular}

\section{(1) \$}

Article Info:

Received: 24 Oct 2020

Accepted: 01 Des 2020

Available Online: 01 April 2021

Keywords:

Breast cancer, Cognitive flexibility, Holographic reprocessing therapy, Posttraumatic growth

\section{A B STRACT}

Background and Aim Breast cancer is among the most prevalent cancers in women with extensive associated biopsychological problems. The present study aimed to determine the effects of holographic reprocessing therapy on cognitive flexibility and posttraumatic growth in women with breast cancer. Methods \& Materials This was a quasi-experimental study with pretest-posttest and a control group design. The statistical population included all patients with breast cancer who underwent mastectomy and who were being treated in the chemotherapy ward of Imam Khomeini Hospital in Ardabil City, Iran, in 2020.

By convenience sampling method, 30 women with breast cancer were selected and randomly divided into the experimental and control groups ( $n=15$ /group). The experimental group, while receiving pharmacotherapy, collectively underwent holographic reprocessing therapy in 9 one-hour sessions (two sessions per week) for 5 weeks; however, the control group received only pharmacotherapy. Both research groups were assessed in pretest and posttest phases by the Cognitive Flexibility Inventory (Dennis \& Vander Wal, 2010) and the Posttraumatic Growth Inventory (Tedeschi \& Calhoun, 1996). Finally, the obtained data were analyzed using Multivariate Analysis of Covariance (MANCOVA) in SPSS v. 23.

Ethical Considerations All study subjects provided a signed written informed consent form. The Research Ethics Committee of Ardabil University of Medical Sciences approved this study (Code: IR.ARUMS. REC.1399.393).

Results The current study results indicated that holographic reprocessing therapy was effective on cognitive flexibility $(\mathrm{F}=42.319, \mathrm{P}<0.01)$ and posttraumatic growth $(\mathrm{F}=18.300, \mathrm{P}<0.01)$ in women with breast cancer; this method also increased their cognitive flexibility and posttraumatic growth.

Conclusion Considering psychological factors and information processing methods in patients with cancer is highly important. Thus, counselors and psychotherapists are advised to use holographic reprocessing therapy as a complementary treatment without adverse effects along with medical treatments to reduce the psychosocial problems caused by the disease.

\section{Extended Abstract}

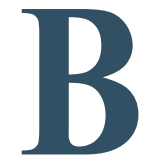

\section{Introduction}

reast cancer targets a symbolic organ in women; thus, it is psychologically, emo- tionally, socially, and sexually more important than other cancers. Accordingly, it can disrupt the regular, daily living activities, making the patient feel that she has lost control of the disease as well as life in general. Therefore, a subject with low cognitive flexibility cannot accept the changes caused by the disease and overcome its challenges [8]. Low

\section{* Corresponding Author:}

\section{Sahar Khoshsorour, PhD. Student}

Address: Department of Psychology, Faculty of Educational Sciences and Psychology, University of Mohaghegh Ardabili, Ardabil, Iran.

Tel: +98 (45) 31505012

E-mail: khoshsorour@uma.ac.ir 
cognitive flexibility could lead to rumination, stress, and ineffective coping strategies when encountering chronic physical pain [9].

Furthermore, experiencing positive psychological changes, known as posttraumatic growth, refers to positive personal and psychological changes that occur after a trauma or traumatic event (being diagnosed with cancer) and results from an individual's struggle against such stressful events [11]. Posttraumatic growth manifests in various forms, including realizing the value of life, making meaningful interpersonal relationships, generating stronger feelings, changing life priorities, and enriching spiritual life [10]. When posttraumatic growth occurs, it can facilitate the process of recognizing and accepting a stressful event in individuals [12].

A method that can be used as a complementary therapy without adverse effects along with medical interventions to reduce cancer-induced psychosocial problems is holographic reprocessing therapy; it is cognitive-experimental psychotherapy, i.e., based on Epstein's cognitive experiential self-theory of personality. In holographic reprocessing therapy, due to applying various techniques that affect cognition, behavior, and emotion, one learns to adopt a better and more effective perception of events [14].

Certainly, considering psychological factors in patients with breast cancer, especially after mastectomy, is among the research priorities. Moreover, the effects of holographic reprocessing therapy remain unexplored on cognitive flexibility and posttraumatic growth in women with breast cancer in Iran. Thus, the present study aimed to determine the effects of holographic reprocessing therapy on cognitive flexibility and posttraumatic growth in women with breast cancer. Accordingly, by paying more attention to cognitive processing in patients and finding manners to correct them, we can develop an effective measure to reduce the secondary problems caused by the disease.

\section{Materials and Methods}

This was a quasi-experimental study with pretest-posttest and a control group design. The statistical population included all patients with breast cancer who underwent mastectomy and who were being treated in the chemotherapy ward of Imam Khomeini Hospital in Ardabil City, Iran, in 2020. Through the convenience sampling method, 30 women with breast cancer were selected and randomly divided into the experimental and control groups $(n=15 /$ group). The inclusion criteria of this study included being diagnosed with breast cancer by an endocrinologist; undergoing mastectomy; being involved with the disease for at least one year; having consent to participate in the study; not consuming any psychiatric medications; being in the age range of 25-55 years, and having a minimum literacy for reading and writing. Exclusion criteria included withdrawal from the study, achieving complete recovery, and presenting acute mental health illnesses.

The experimental group, while receiving pharmacotherapy, collectively underwent holographic reprocessing therapy in 9 one-hour sessions (two sessions per week) for 5 weeks; however, the control group received only pharmacotherapy. Both research groups were assessed in pretest and posttest phases by the Cognitive Flexibility Inventory [23] and the Posttraumatic Growth Inventory [25]. Finally, the collected data were analyzed using Multivariate Analysis of Covariance (MANCOVA) in SPSS.

\section{Results}

The present research results revealed that holographic reprocessing therapy was effective on cognitive flexibility $(\mathrm{F}=42.319, \mathrm{P}<0.01)$ and posttraumatic growth $(\mathrm{F}=18.300$, $\mathrm{P}<0.01)$ in women with breast cancer; this approach also increased their cognitive flexibility and posttraumatic growth.

\section{Discussion and Conclusion}

The current study determined the effects of holographic reprocessing therapy on cognitive flexibility and posttraumatic growth in women with breast cancer. Notably, holographic reprocessing therapy improves' clients adjustability by modifying cognitive processing and the reassessment of their beliefs. In this method, the therapist teaches the clients various coping strategies; subsequently, they can replace these coping skills with previous ineffective methods in stressful situations. This measure requires improved cognitive flexibility. Additionally, by drawing an experimental hologram, the therapist recreates the experience of managing the emotions caused by trauma and disease; clients face the emotions instead of avoiding them and find more logical and meaningful justifications for them while expressing emotions [27]. This is an exercise in cognitive flexibility. Finally, this method emphasizes the establishment of new communication patterns and the formation of new goals for the future; they can be achieved by the reinforcement of cognitive flexibility.

Spontaneous and disturbing thoughts are often uncontrolled and indicate a stress response to the traumatic event. Moreover, they stand against controlled, more positive, and purposeful thoughts. Consequently, clients, during therapy sessions, learn to adopt further control over their thoughts. This is because the deliberate and purposeful reprocessing of trauma might reduce the level of anxiety. Such condi- 
tions could also increase the acceptance of emotions, and the potential for posttraumatic growth. This ultimately increases the degree of control of dysfunctional negative thoughts and leads to habituation instead of avoiding the thoughts and subsequent emotions [28]. The present study data can have implications for mental health professionals. By providing educational programs on mental health and paying more attention to information processing procedures in patients, one can reduce the pathogenicity of this disease and prevent its subsequent consequences.

\section{Ethical Considerations}

\section{Compliance with ethical guidelines}

All study subjects signed the written informed consent form. The Research Ethics Committee of Ardabil University of Medical Sciences approved this study (Code: IR.ARUMS.REC.1399.393).

\section{Funding}

This research received no specific grant from funding agencies in the public, commercial, or not-for-profit sectors.

\section{Authors' contributions}

All authors met the standard criteria of writing based on the recommendations of the International Committee of Medical Journal Publishers (ICMJE).

\section{Conflicts of interest}

The authors declared no conflicts of interest. 


\title{
اثربخشى درمان يردازش مجدد هولوترافيك بر انعطافيذيرى شناختى و رشد يس از سانحه در زنان مبتلابه سرطان سينه
}

\author{
سيفاله آقاجانى' هـ •سحر خوشسرور 'ه سارا تقىزاده هير'ه
}

1. كروه روانشناسى، دانشكده علوم تربيتى و روانشناسى، دائشكاه محقق اردبيلى، اردبيل، ايران.

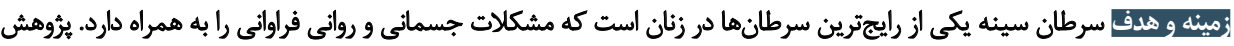

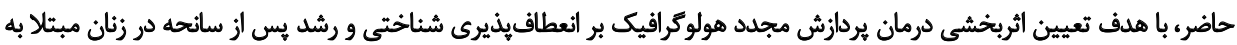
سرطان سينه انجام شد.

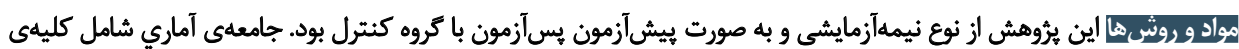

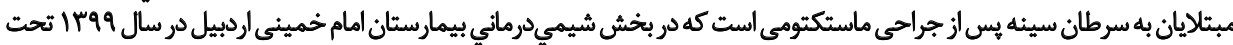

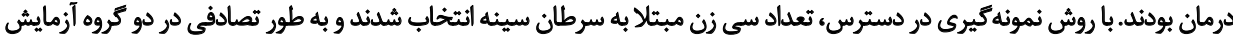

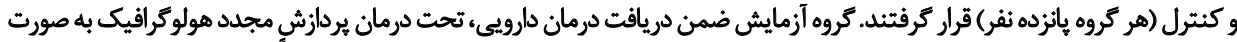

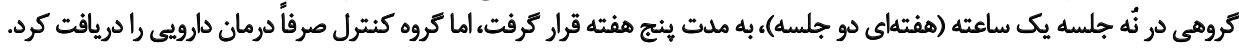

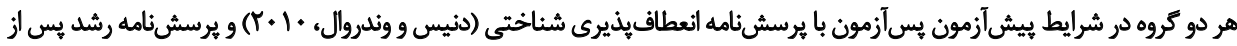

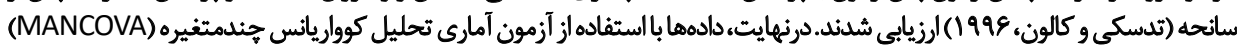

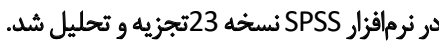

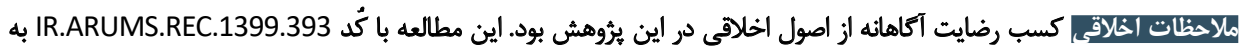

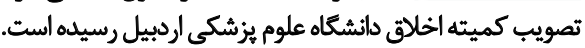

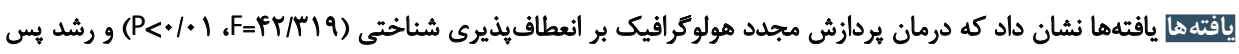

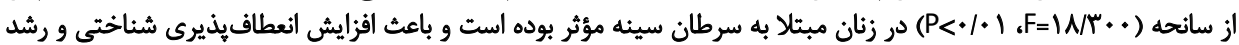

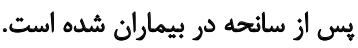

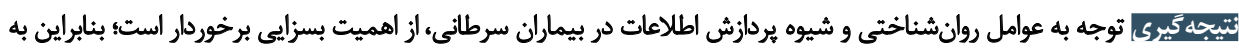

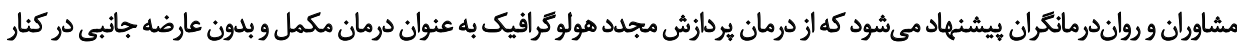

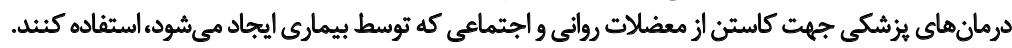

خانوادكى، تمايلات جنسى، كار و مراقبت از خود اختلال ايجاد

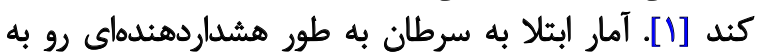

مقدمه

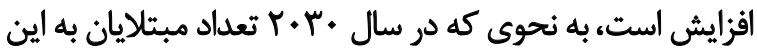

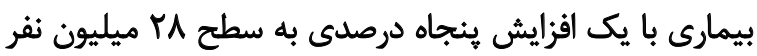

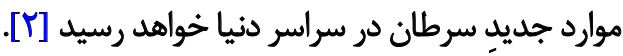
سرطان سينه' يكى از شايعترين انواع سرطان در زنان است

سرطان، يكى از انواع كوناكون بيمارىهاى مزمن است و

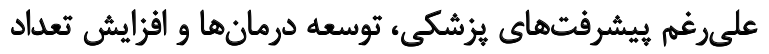

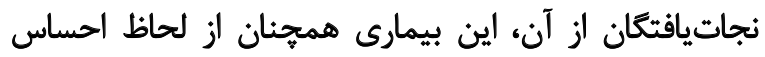

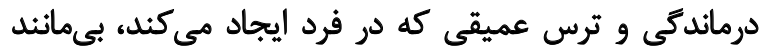

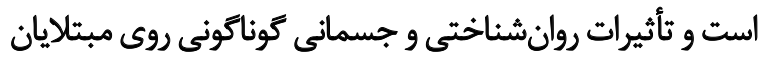

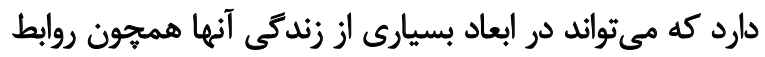


استرس و راهبردهاى مقابلهاى ناكارآمد در مواجهه با دردهاى

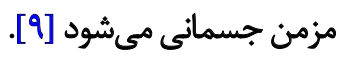

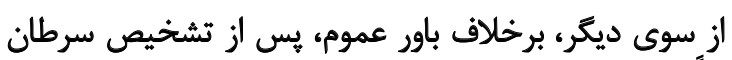

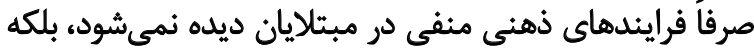

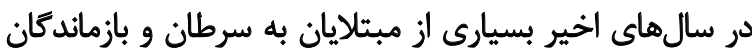

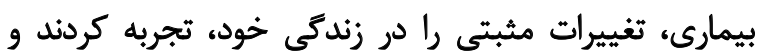

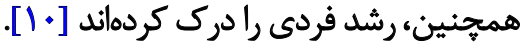
تجربهى تغييرات روانشناختى مثبت كه از آن تحت

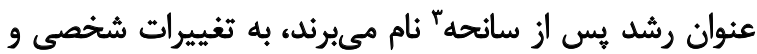

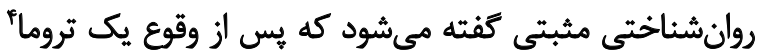

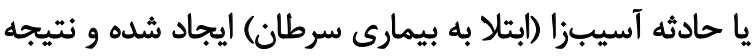

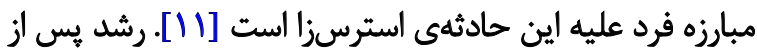

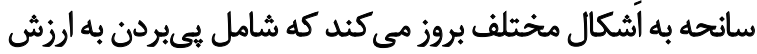

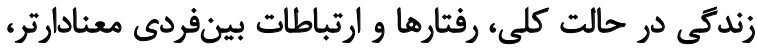

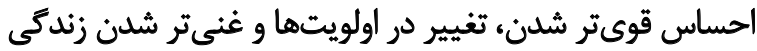

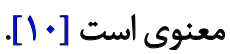

شناخت مفهوم رشد يس از سانحه، اطلاعانى در اختيار فعالان

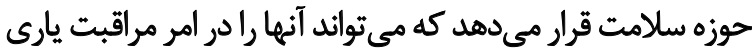

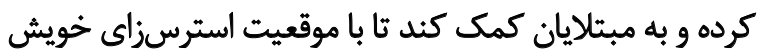

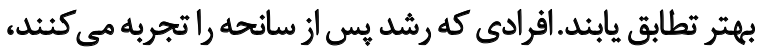

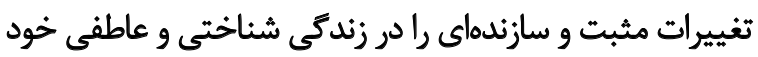

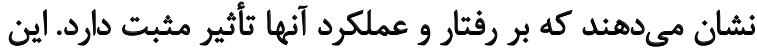

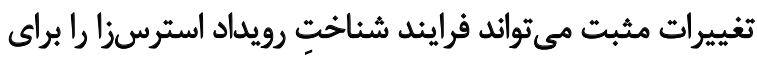

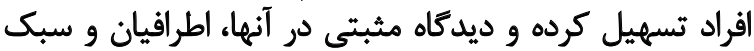

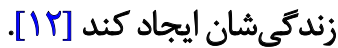

زنان مبتلا به سرطان سينه از ابتداى تشخيص، يريشانى

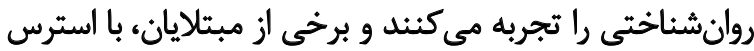

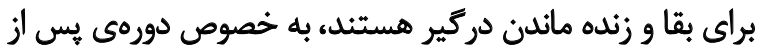

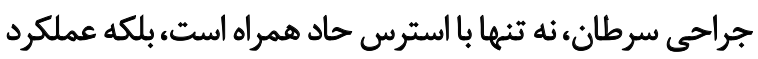

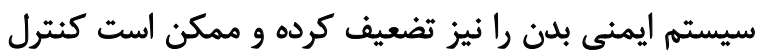

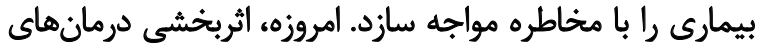

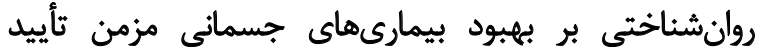

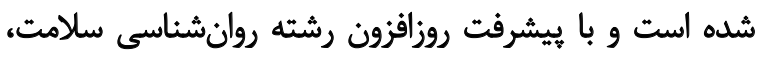

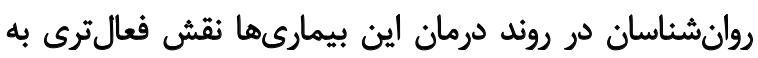

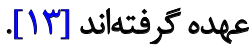

روش درمان يردازش مجدد هولوكرافيكه نيز يك شئيوه

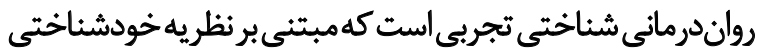

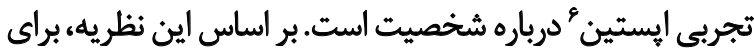

\footnotetext{
3. Posttraumatic Growth

4. Trauma

5. Holographic Reprocessing Therapy

6. Epstein Cognitive- experiential Self-theory
}

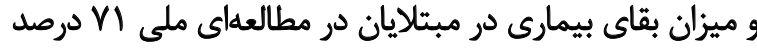

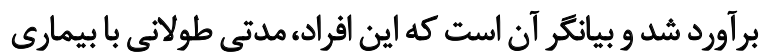

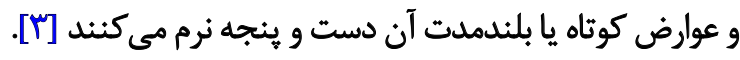
سرطان سينه، زنان بسيارى رادر سنين كار و يرورش خانواده،

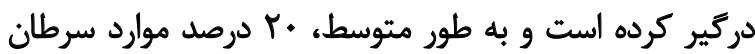

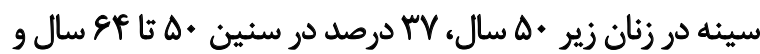

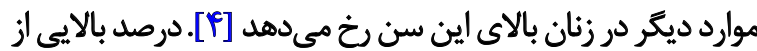

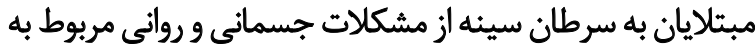

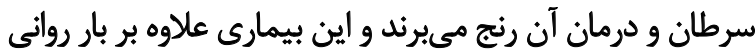

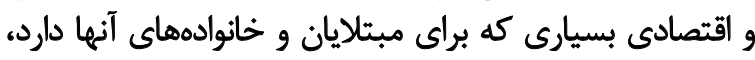

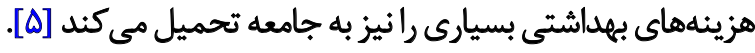
وقتى فرد با تشخيص سرطان روبهرو مي شوده، معمولاً افكار و

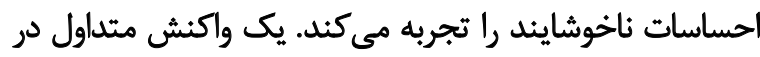

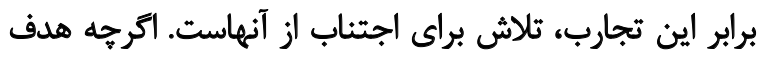

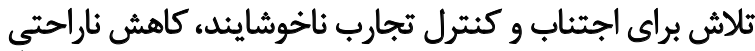

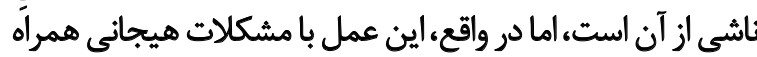

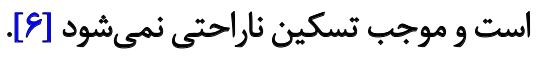

افراد مبتلا به سرطان با كاهش دركيرى در فعاليتهاى

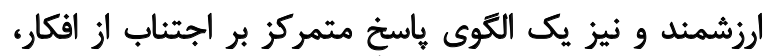

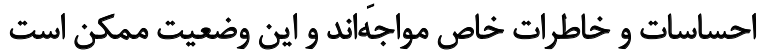

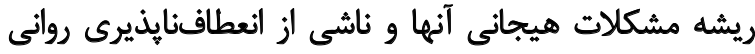

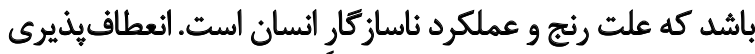

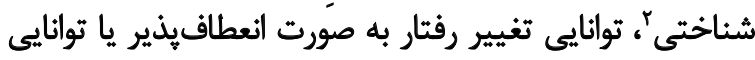

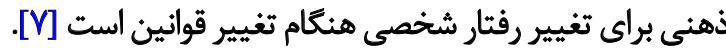
رنج وقتى رخ مى دهد كه ثلاش فرد براى تجزيه و تحليل

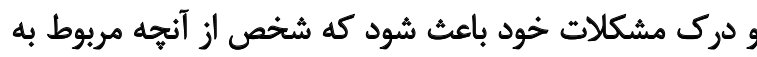

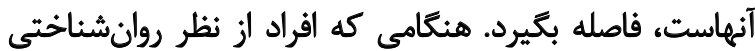

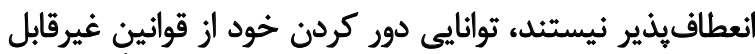

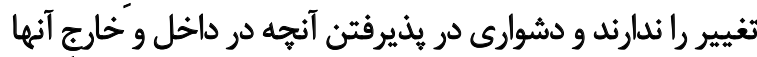

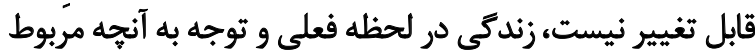

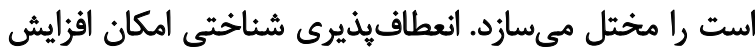

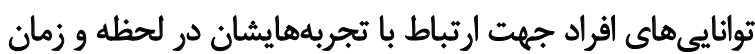

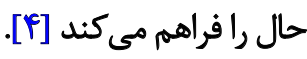

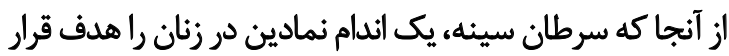

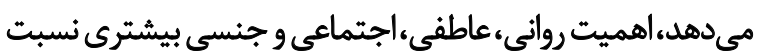

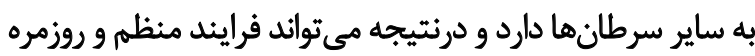

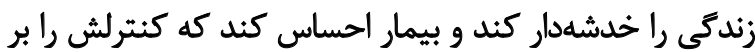

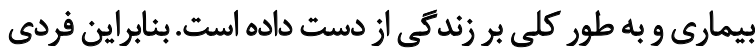

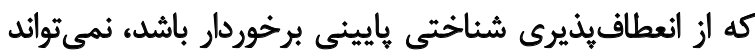

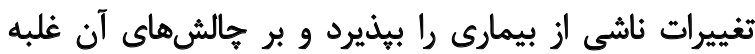

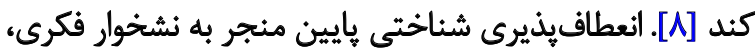

2. Cognitive Flexibility 
شناختي و رشد يس از سانحه در زنان مبتلا به سرطان سينه

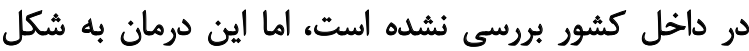

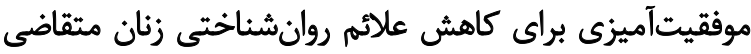

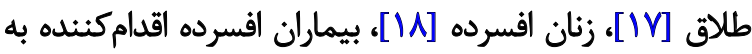

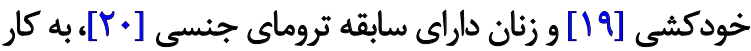

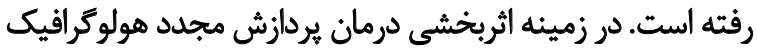

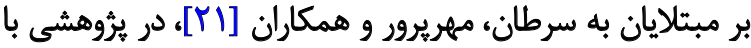

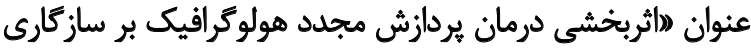

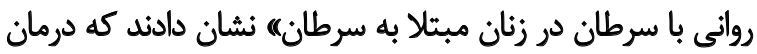

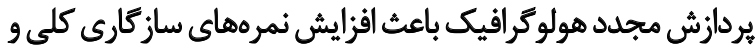

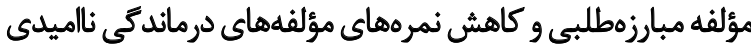

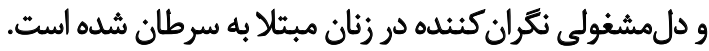

از يك سو، سرطان سينه يكى از رايجترين سرطانها در

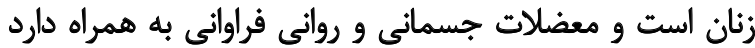

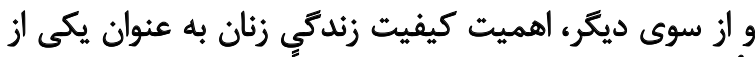

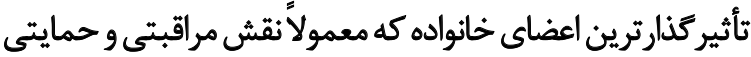

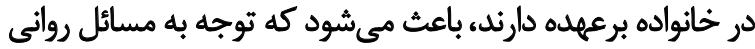

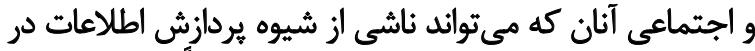

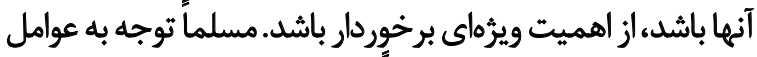

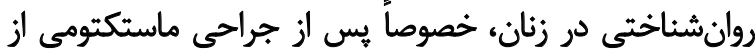

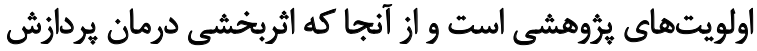

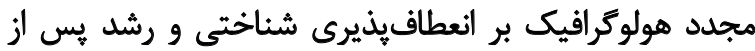

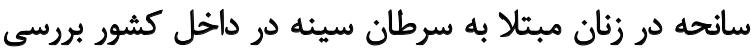

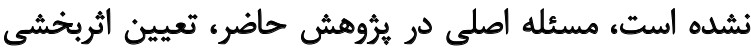

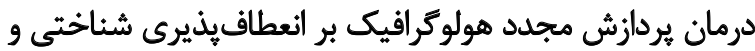

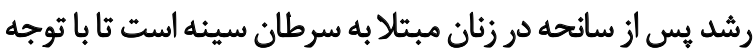

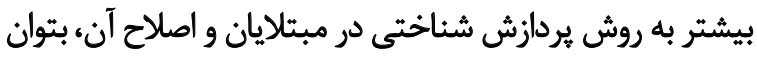

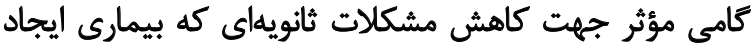
مي كند، برداشت. مؤتر جتش.

مواد و روشها

اين برؤهش از نوع طرحهاى نيمهآزمايشى و به صورت

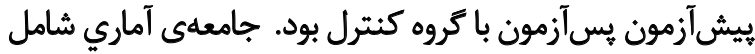

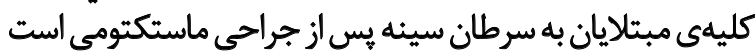

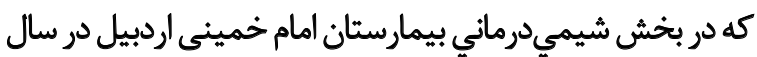
99 1 آتحت درمان بودند.

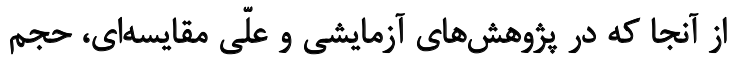

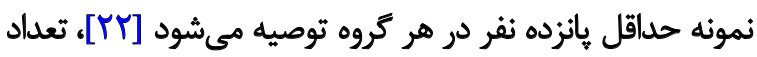

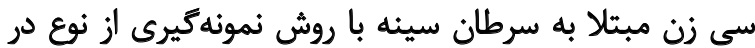

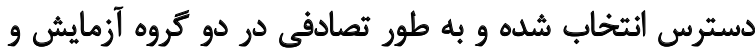

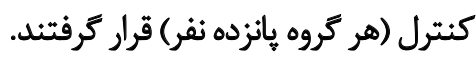

ملاكهاى ورود در اين يُروهش، تشخيص سرطان سينه از

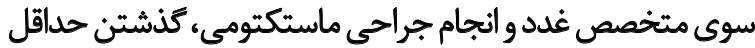

يردازش اطلاعات دو سيستم مجزاي عقلاتى و وتجربى وجود

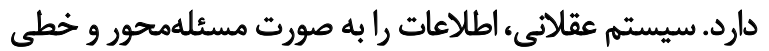

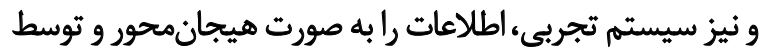

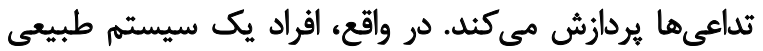

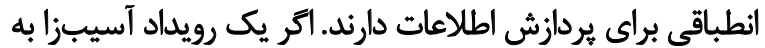

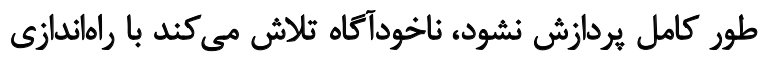

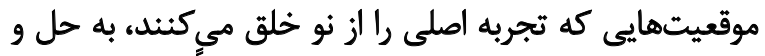

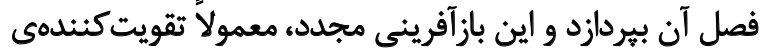

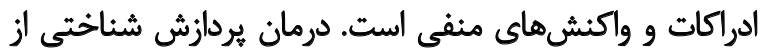

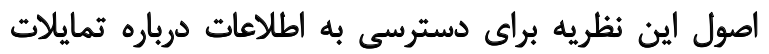

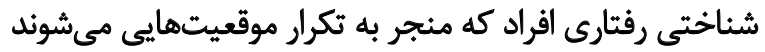

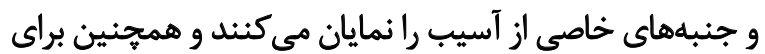

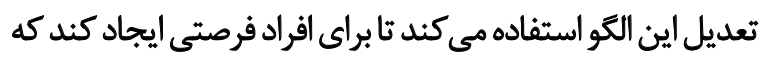

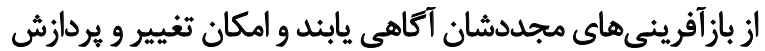

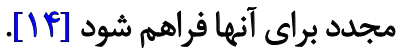

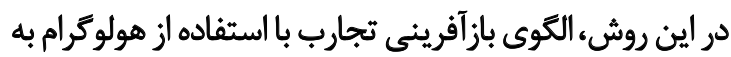

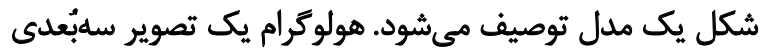

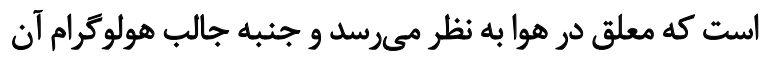

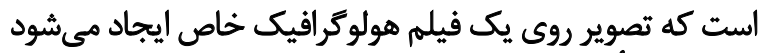

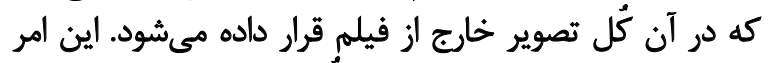

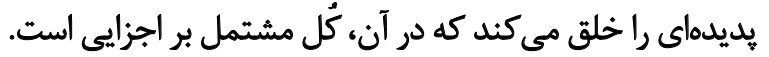

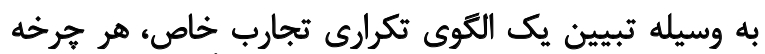

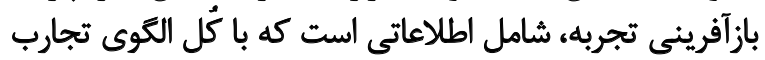

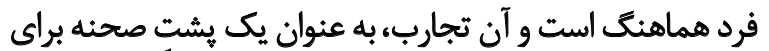

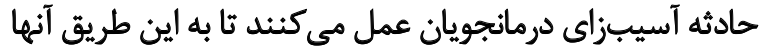

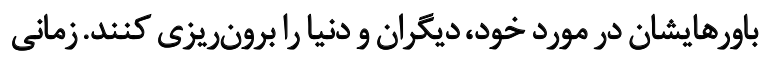

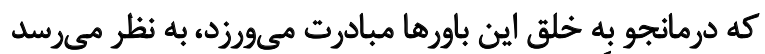

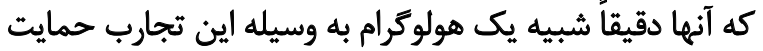

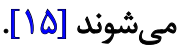

به عبارت ديكر، تجربه بديدهاى هولوكرافيك است و به اين دليل

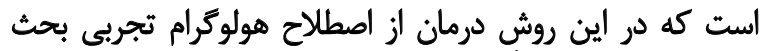

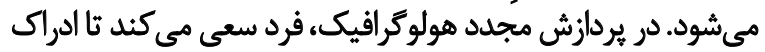

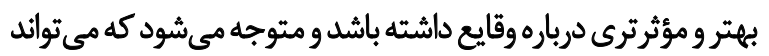

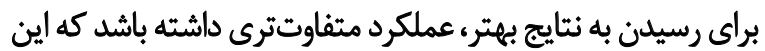

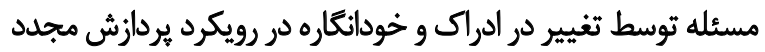

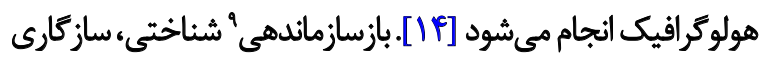

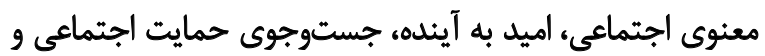

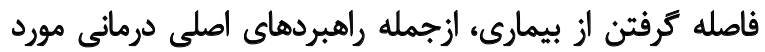

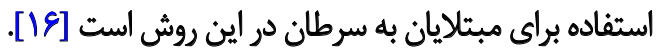
اثربخشى درمان يردازش مجدد هولوكر افيك بر انعطافيذيرى

\section{Rational}

8. Experiential

9. Reorganization 


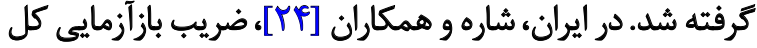

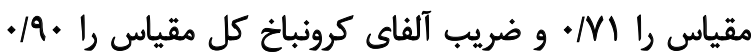

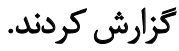

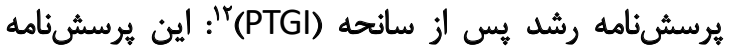

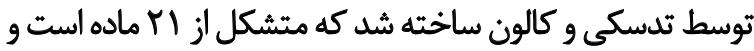

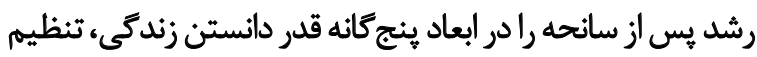

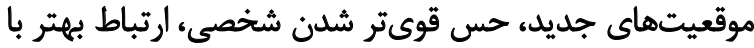

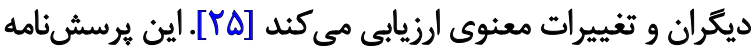

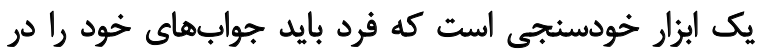

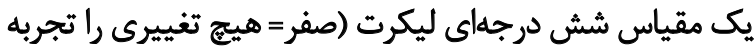

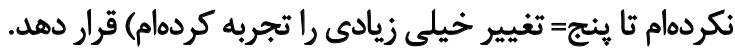

دامنه نمرات بين صفر تاه • ا است و كسب نمرات بالاتر نشانكر

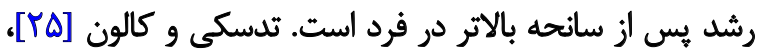

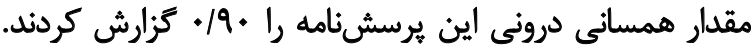

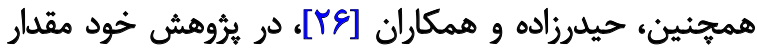

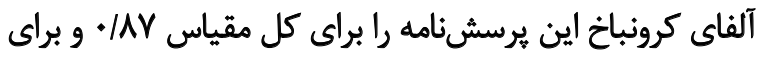

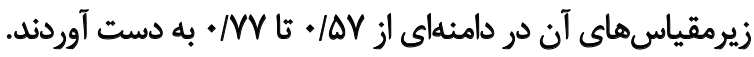
در اين يُروهش، يروتكل درمانى طبق راهنماي درمانى كاتز

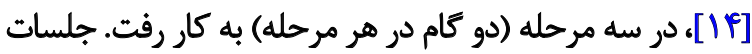

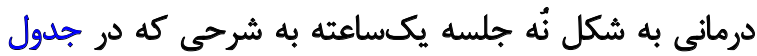
شماره ا آمده است، اختصاص داده شد.

ياقتهها

بررسى ويرُخى هاى جمعيتشناختى نشان داد كه ميانكين و انحراف استاندارد سنى براى كروه آزمايش

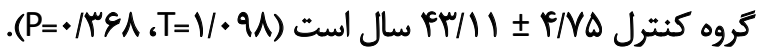

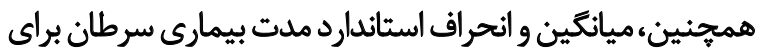

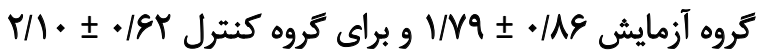

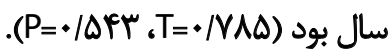

در اين يُؤهش به منظور تعيين اثربخشى درمان يردازش

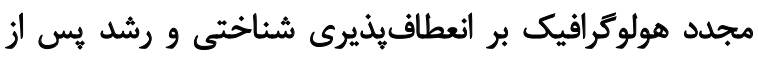

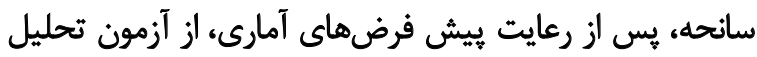

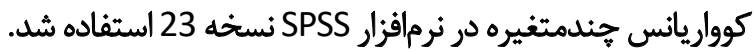

با بررسى آزمون ام باكس مشخص شد مفروضه همكنى مائريس

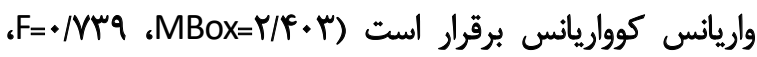

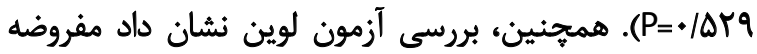

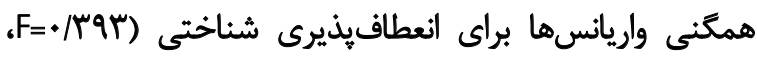

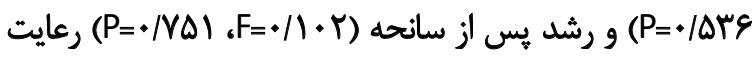

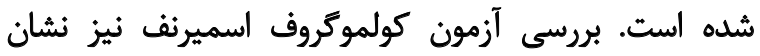

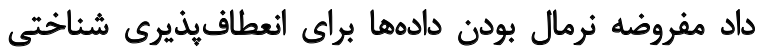

12. Posttraumatic Growth Inventory (PTGI)

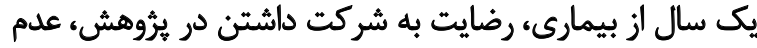

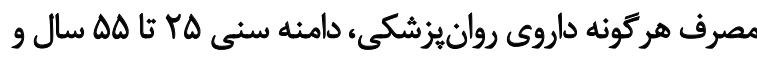

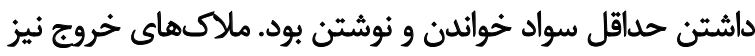

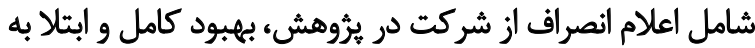
بيمارىهاى حاد روانى بودئل.

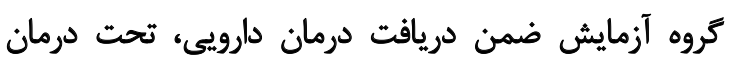

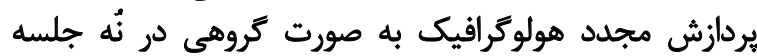

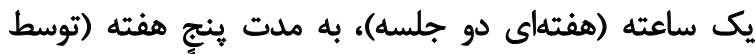

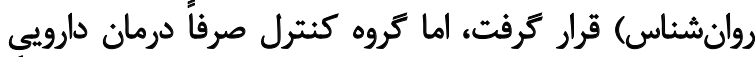

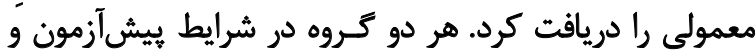

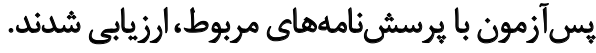
در اين يُروهش، از تمامى افراد شركت كنينده، رضايت درمانى

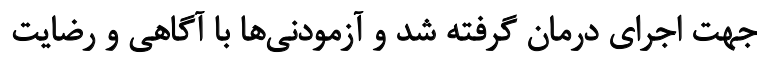

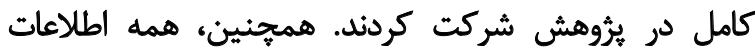

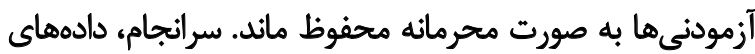

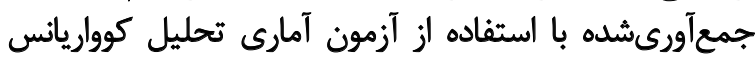

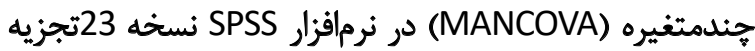

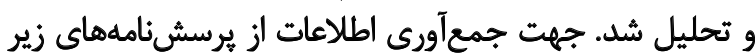

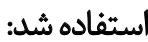

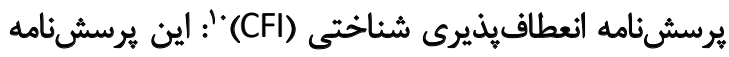

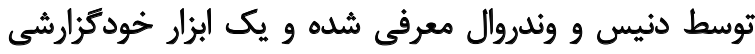

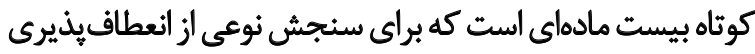

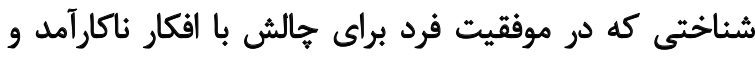

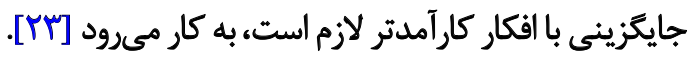

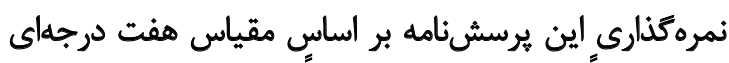

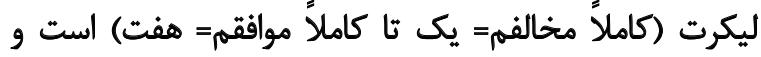

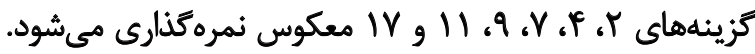

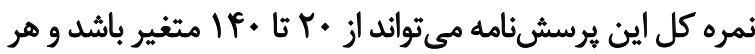

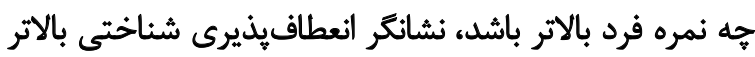

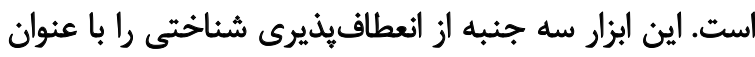

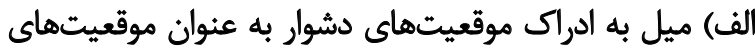

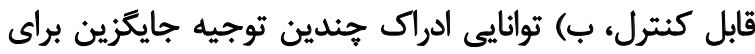

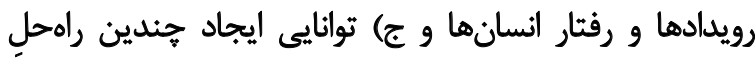
جايكزين براى موقعيتهاى دشوار مى رسنجد.

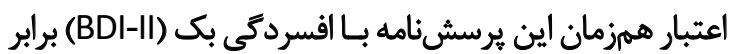

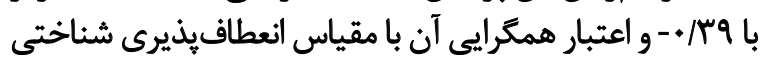

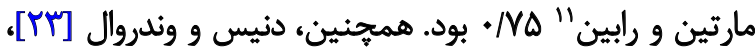

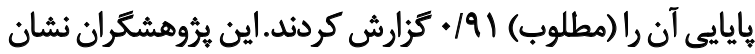

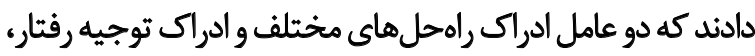

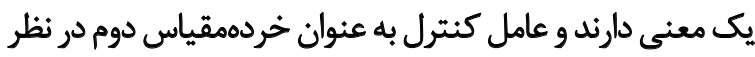

10. Cognitive Flexibility Inventory (CFI)

11. Martin \& Rubin 
جدول ا. محتواي جلسات درمان يردازش مجدد هولوكرافيك

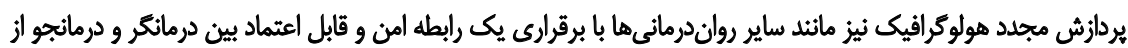

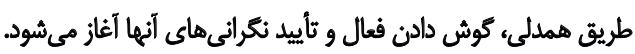

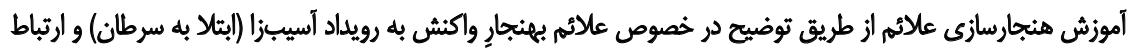

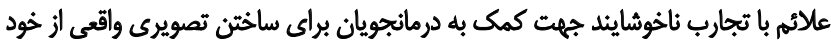

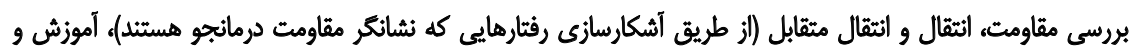
تفكيك دو سيستم عقالاني و هيجاني

آموزش مهارتهاى مقابلهاى، فنون آرامش آموزى و افزايش خودكارآمدى همجحون تكنيكهاى آرامش آموزى و يسخورانداند

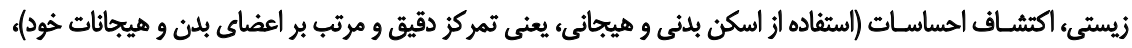

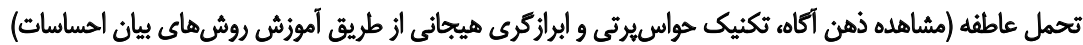
أموزش ثاب آورى و انعطاف بذئيى هيجانى (الز طريق افزايش شوخ طبيى و كسب حمايت الجتماعى)، آموزش ايجاد شبكه

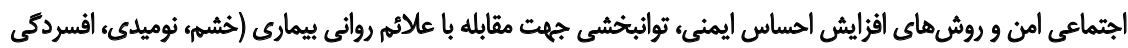

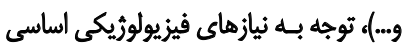

مراخدتن به اكتشاف تجربي از طريق ارتباط برقرار كردن با تجربه ناخوشايثد و شناسايي احساسات ناخوشايند ناشى از بيمارى،

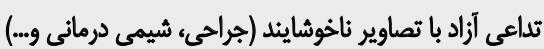

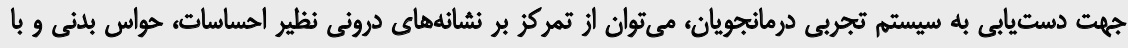

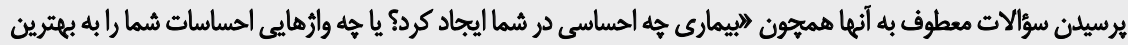
نحو توصيف مى كند؟

جهت ترسيم هولوكرام تجربى درمانجويان از تمثيل ساده كترى روى بخارى 'استفاده شد. درمانجويان با استفاده از اين تمثيل،

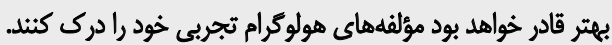

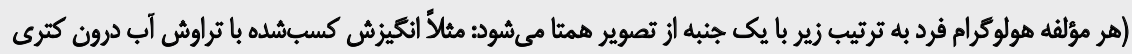

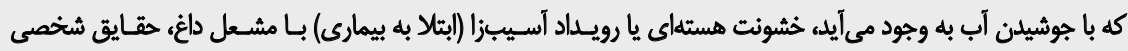

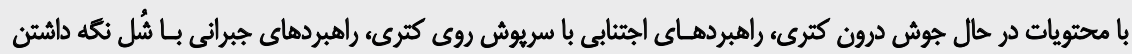

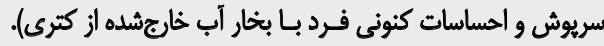

\section{كام يك: برقرارى}

اتحاد درمانى

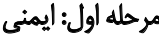
(جلسات اول، دوم، مرحله

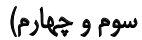

كام دو: ثأمين

خار خجوب مهارتهاي مقابلهاى

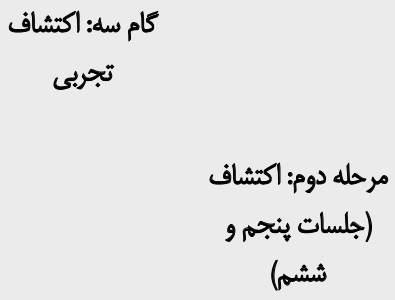

كام جهار: ترسيم هولوكرام تجرئي

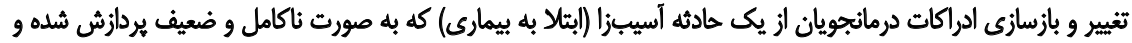

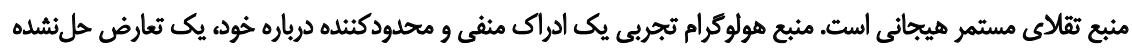

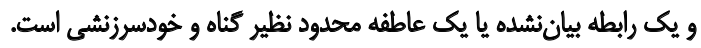

به طور خلاصه، در كامهاي يرداز ش مجلده با اجازه درمانجو روى رويداد أسيبزا تمركز شد (دريافت تشخيص سرطان، شيمى

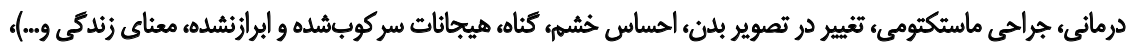

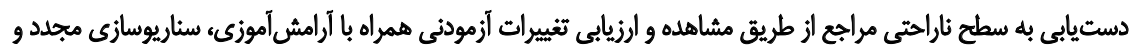

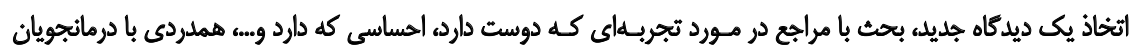

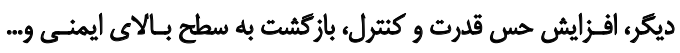

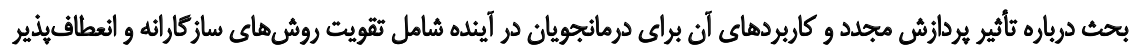

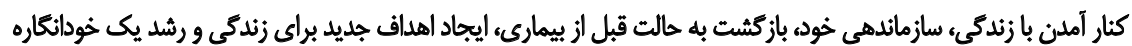

كام ينج: بودازش

مجدد هولوكرام

تجربى

$$
\begin{aligned}
& \text { مرحله سوم: } \\
& \text { يبردازش مجدد } \\
& \text { (جلسات هفتم، } \\
& \text { هشتم ونهم) }
\end{aligned}
$$

كام ششش: ايجاد

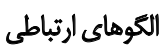

جليد 
جدول r. شاخصهاي توصيفى در متغيرهاى يُروهش در كروه آزمايش و كثترل

\begin{tabular}{|c|c|c|c|c|}
\hline \multicolumn{4}{|c|}{ ميانكين土|نحراف معيار } & \multirow{3}{*}{ متغير } \\
\hline \multicolumn{2}{|c|}{ كروه كتترل } & \multicolumn{2}{|c|}{ كروه آزمايش } & \\
\hline يس آزمون & يبشأزمون & بس آزمون & ييش أزمون & \\
\hline $9 . / \& 9 \pm+r / 8 \Delta$ & $91 / M^{\top} \pm \Delta / 11$ & $q \varepsilon / \& \varepsilon \pm \Delta / T \gamma$ & $91 / 4 \cdot \pm 4 / \% \wedge$ & انعطافيذيرى شناختى \\
\hline$V \cdot / 4 \varepsilon \pm f / \lambda$. & $\varepsilon q / \cdot \varepsilon \pm F / A r$ & $V / / \varepsilon+ \pm \Delta / \pi f$ & ENTE $\pm \Delta / T T$ & رشد يس از سانحه \\
\hline
\end{tabular}

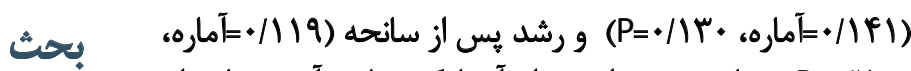

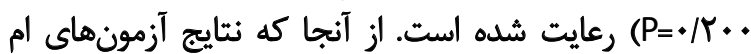

تحليل يافتههاي اين يُوهش و نتايج حاصل از آزمون تحليل

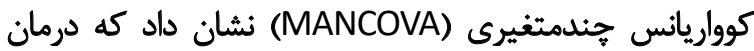

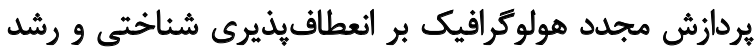

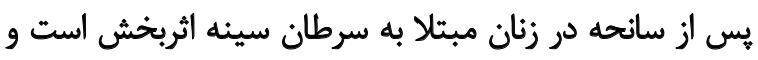

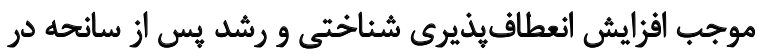
مبتلايان به سرطان سينه مى مئود.

يافتهاى يثوهش حاضر مبنى بر اثربخشى درمان بردازش

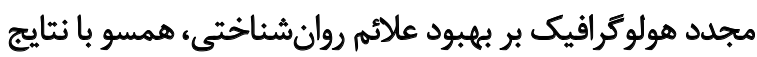

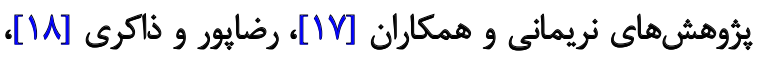

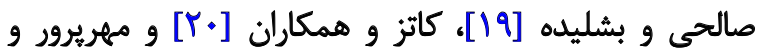

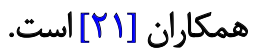

در تبيين اثربخشى درمان يردازش مجدد هولوخرافيك بر براني

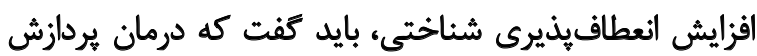

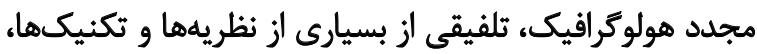

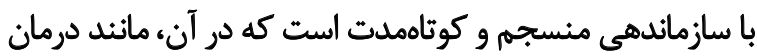

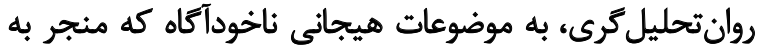

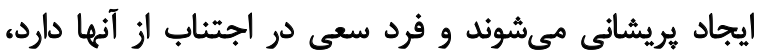

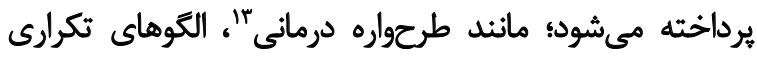
شناسايي مىشوند و مانيد درمان شناختى رفتاري، اين الكوها

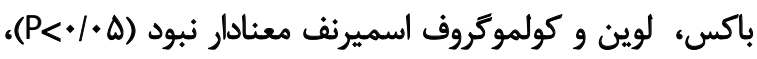

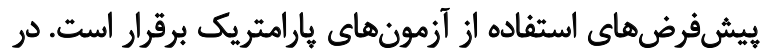

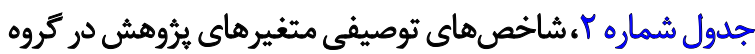

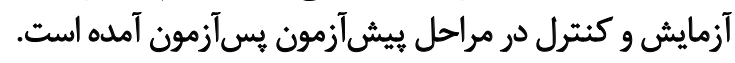
همانطور كه در جدول شماره ب مشاهده مي شود، تمامى

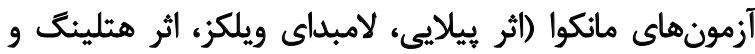

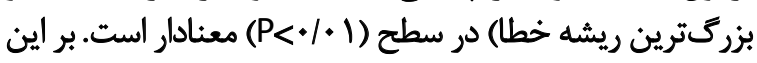

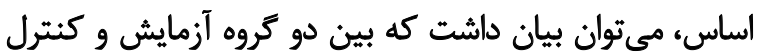

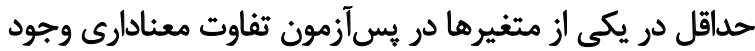

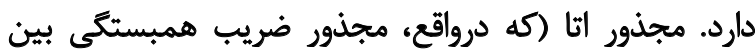

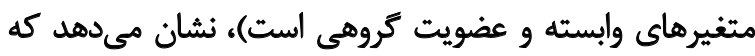

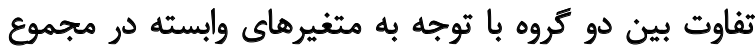

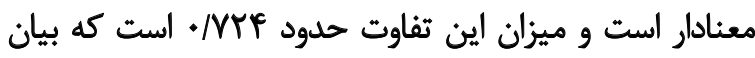

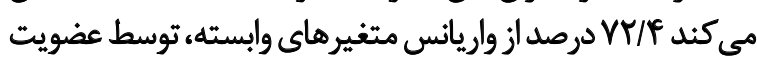

$$
\text { كروهى تبيين شده است. }
$$

جدول شماره F نشان داد در مرحله يس آزمون، بين دو كروه

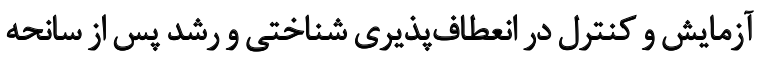

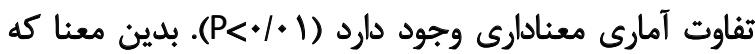

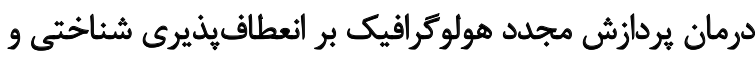
رشد يس از سانحه در زنان مبتلا به سرطان سينه مؤثر بوده است.

13. Schema Therapy

جدول ب. نتايج حاصل از تحليل كوواريانس جندمتغيره نمرات انعطاف يذيرى شناختى و رشد پيس از سانحه

\begin{tabular}{|c|c|c|c|c|c|c|c|}
\hline توان أمارى & مجنور اتا & سطح معنادارى & حُطاى درجه آزادى & فرضيه درجه آزادى & توليائيل & مقدار & ناهم آزهون \\
\hline $1 / \ldots$ & . MTF & $.1+1$ & To & r & MT/ANT & - Mrt & اثر ييلايي \\
\hline $1 / \cdots$ &.$M T F$ & $*+.1$ & TA & r & MT/AMf &.$/ T V E$ & لامبداى ويلكز \\
\hline $1 / \ldots$ & . MTE & $.1 . .1$ & TA & r & MT/AME & TIETY & اثر هثلينك \\
\hline $1 / \ldots$ &.$M T r$ & $+1+.1$ & Ta & $r$ & MT/ATf & T/gTV & بزركترين ريشه خطا \\
\hline
\end{tabular}


جدول F. نتايج حاصل از تحليل مانكوا بر نمرات انعطاف يذيرى شناختى و رشد يس از سانحه در يَسآزمون

\begin{tabular}{|c|c|c|c|c|c|c|c|}
\hline توان آمارى & مجذور اتا & سطح معنادارى & تحليل واريانس & ميانغين مجذورات & درجه آلزادى & مجموع مجذورات & متغير \\
\hline Vo. & .1819 & $.1+\infty 1$ & TT/MIQ & PAEM+P & 1 & resp+r & انعطاف يذيرى شناختى \\
\hline - lut &.$/ P i r$ & $.1 . .1$ & Wr... & $1.9 / 181$ & 1 & $1.9 / 1 \& 1$ & رشد يس از سائحه \\
\hline
\end{tabular}

در مراحل بعدى، به منظور آموزش مهارتهاى مقابلهاي،

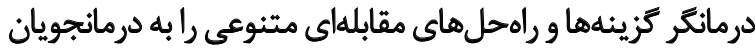

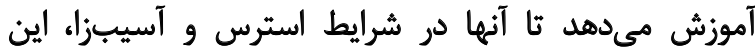

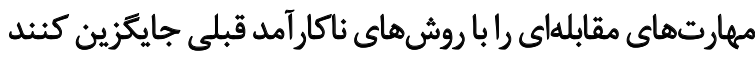

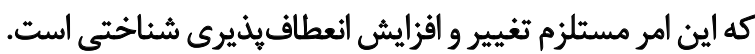

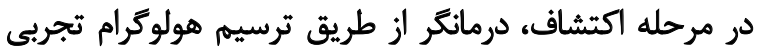

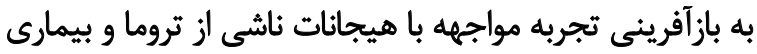

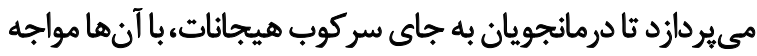

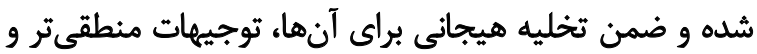

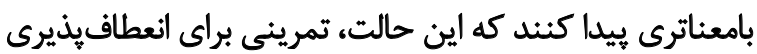
شناختى الست.

درنهايت، در مرحله يردازش مجدد، اهدافي از قبيل برقرارى

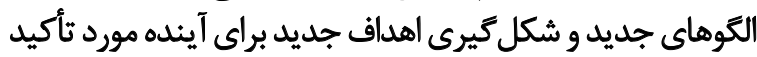

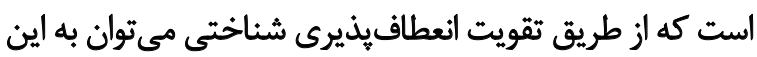

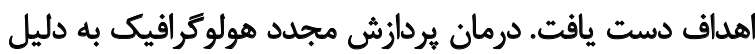

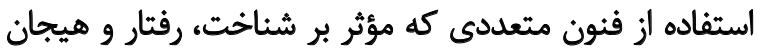

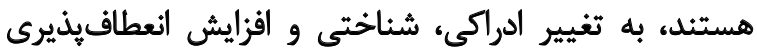

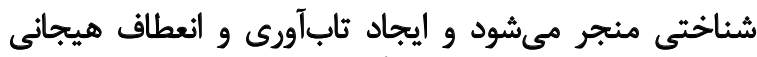

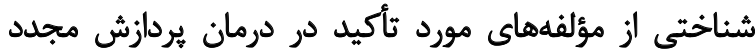

هولوكرافيك است [YV]

جهت تبيين اثربخشى درمان يردازش مجدد هولوزرافيك بر

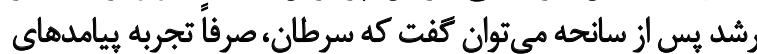

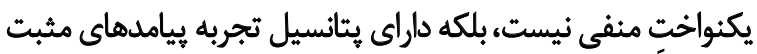

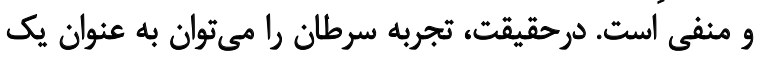

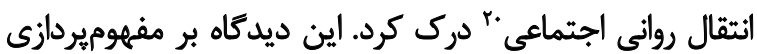

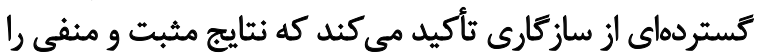

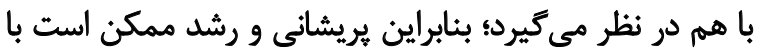

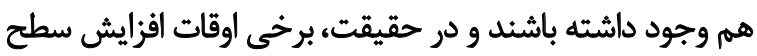

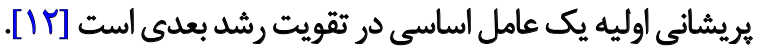

يك تجربهى تروماتيك (مثل تجربه ابتلا به بيمارى سرطان)،

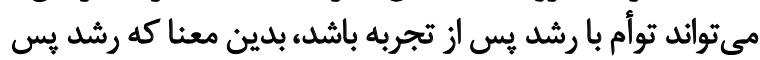

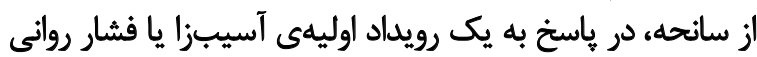

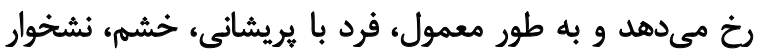

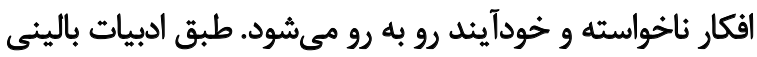

20. Psychosocial Transition
با استفاده از تكنيكهاى مبتنى بر شناخت (همجون بازآرايى)

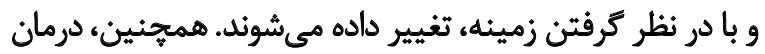

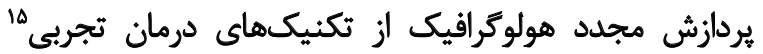

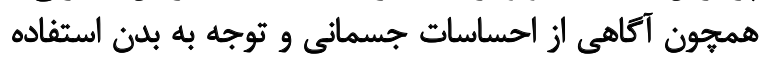

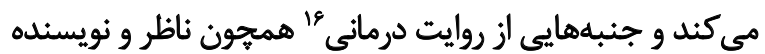

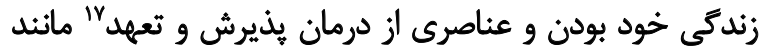

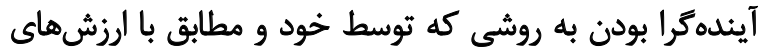

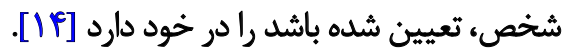

درمان بردازش مجدد هولوكرافيك، تأثير كلى ثروما يا حادثه

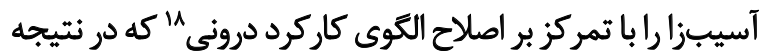

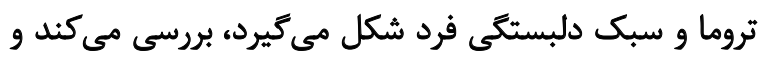

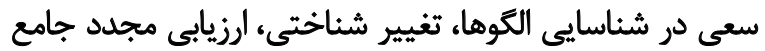

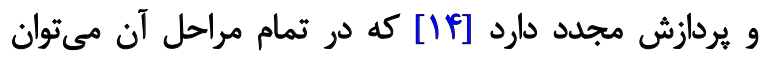

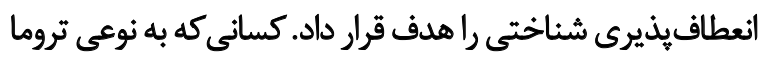

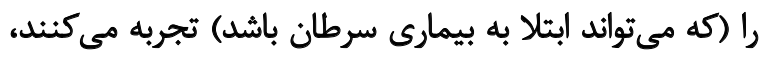

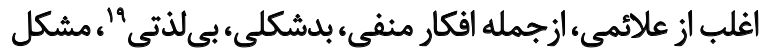

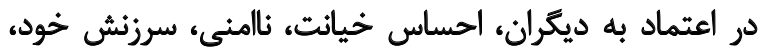

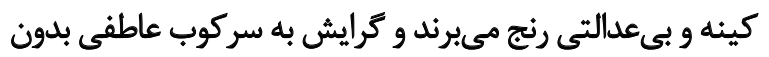

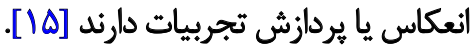

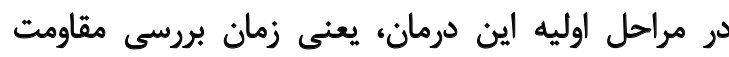

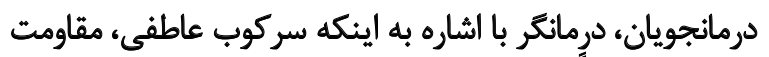

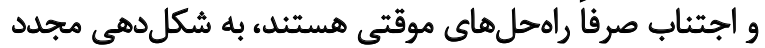

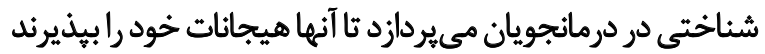

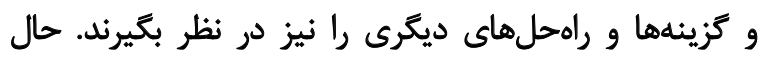

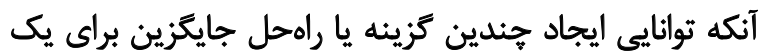

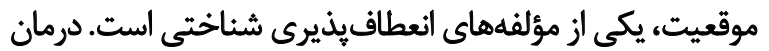

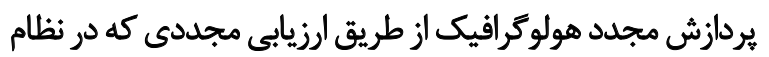

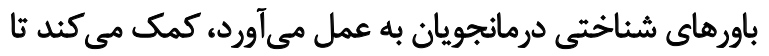

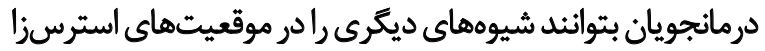

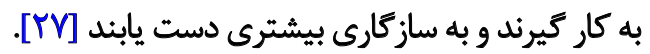

14. Reframing

15. Experiential Therapy

16. Narrative Therapy

17. Acceptance and Commitment Therapy (ACT)

18. Internal Working Model

19. Anhedonia 
است و مردم عادى هيج نوع بيمارى حاد يا مزمنى را به اندازهى

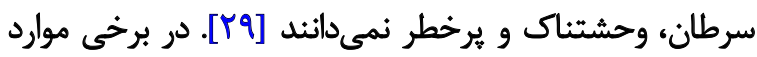

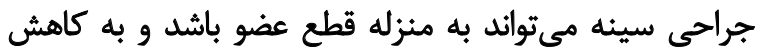

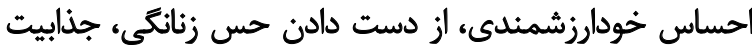

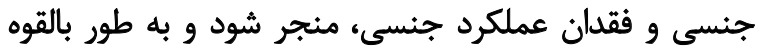

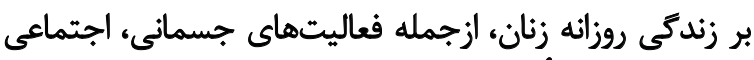

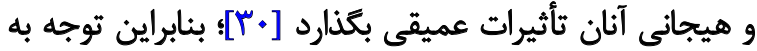
مسائل روانشناختى مبتلايان ضرورى به نظر مىرسد.

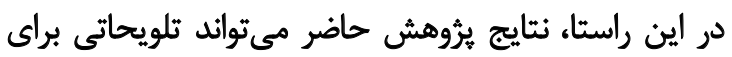

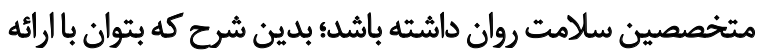

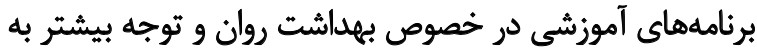

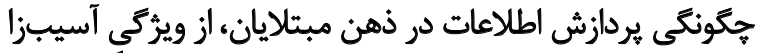

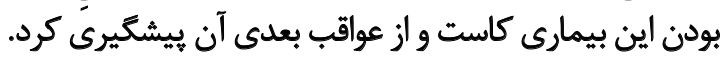

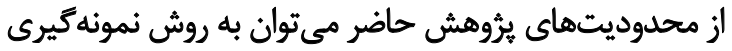

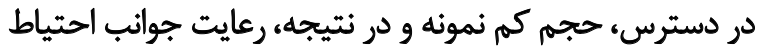

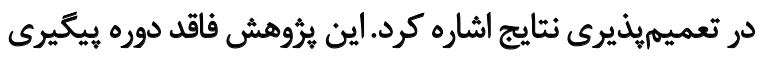

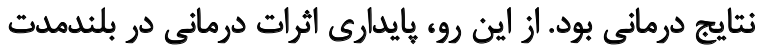

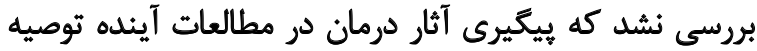

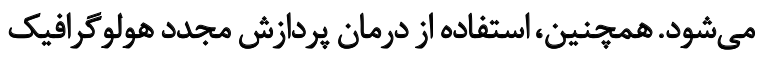

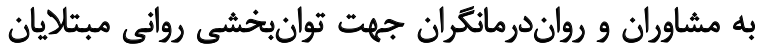

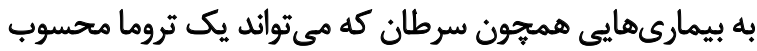
شود، يويشنهاد مي شود. نتيجلميرى

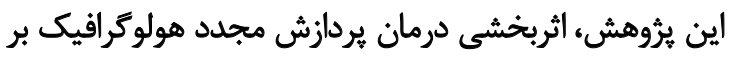

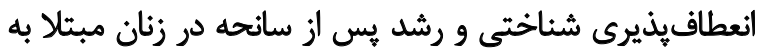

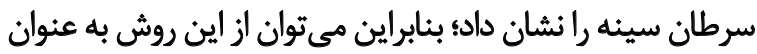

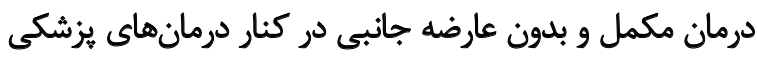

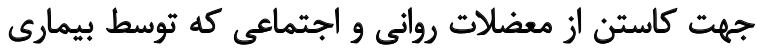

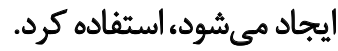

\section{مالاحظات اخلاقي ت م}

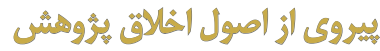

اين مقاله داراى كد اخلاق

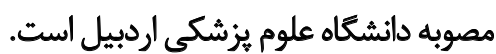

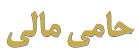

اين ثيروهش، هيج كمك مالى خاصى از سازمانهاي تأمين

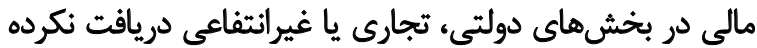
السيت.

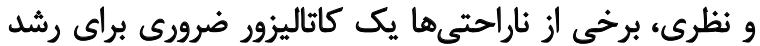

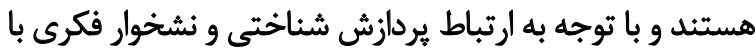

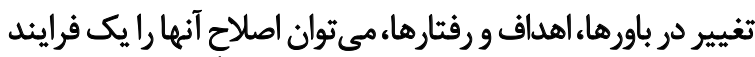

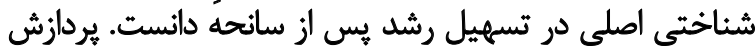

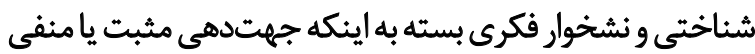

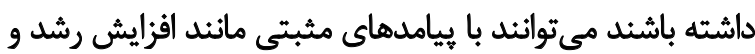

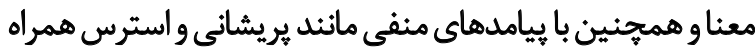

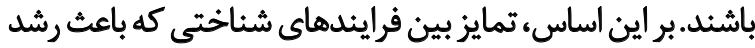

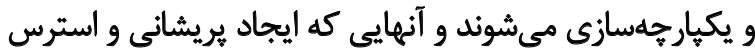

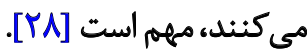

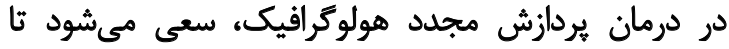

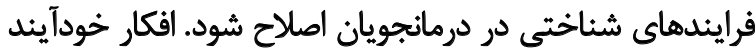

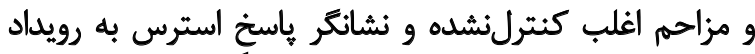

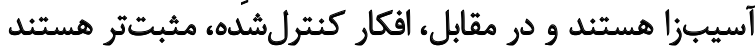

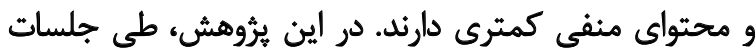

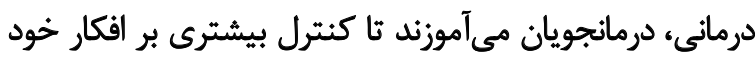

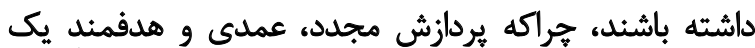

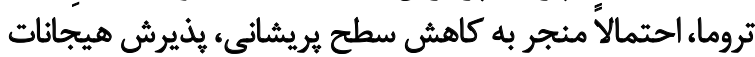

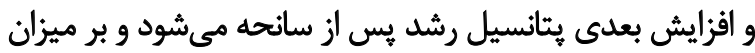

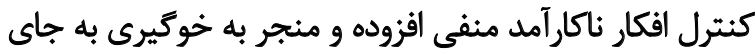
اجتناب از آنها و هيجانات متعاقب مي مئشود.

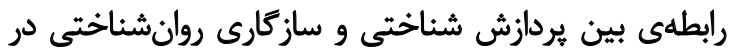

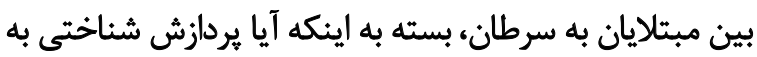

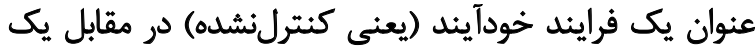

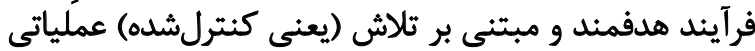

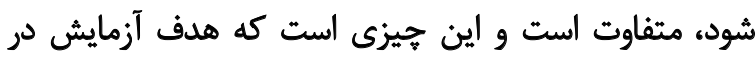

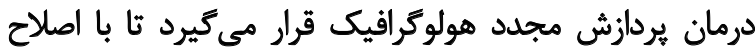

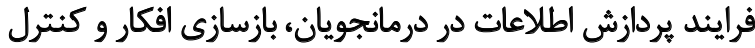

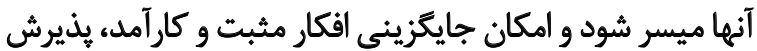
احساسات و درك ارزشمندى زندكى فرانئ فراهم شود. اغلب، در رشد بس از سانحه، سه حوزه تغيير مثبت در افراد

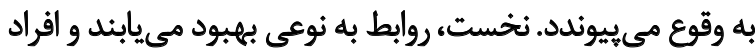

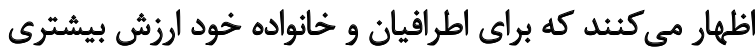

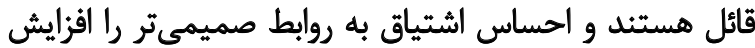

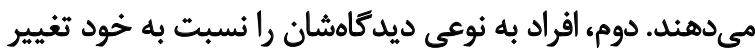

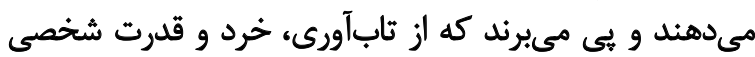

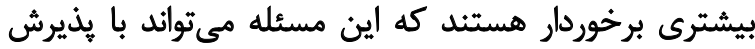

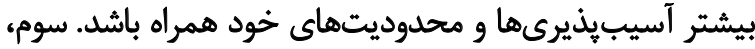

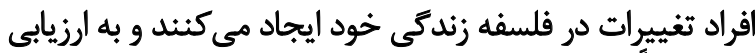

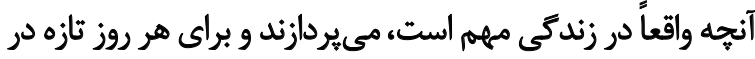

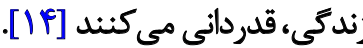

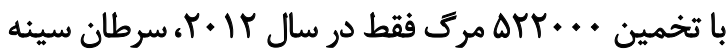
اصلىترين علت مرك بر اثر سرطان در زنان در سرال سراسر جهان سرطين 


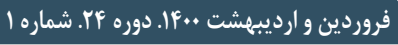

每

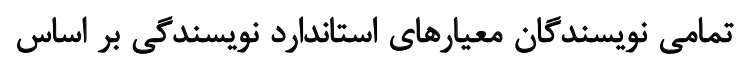

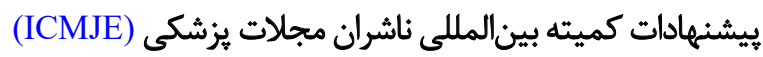
را دارا بودند.

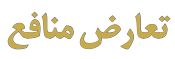

هيجّونه تضاد منافعى در نتارش اين مقاله وجود ندارد.

119

سيفاله أقاجانى و همكاران. اثربخشى درمان يرداز ش مجدد هولؤرافيك در زنان مبثلا به سرطلن سينه 


\section{References}

[1] Özkal F, Arıkan Y. The opinions of patients and patients relatives on announcement of the cancer diagnosis to the patient. Glob J Adv Pure Appl Sci. 2014; 4:179-83. http://archives.un-pub.eu/index.php/ paas/article/viewArticle/3280

[2] World Health Organization (WHO). Cancer [Internet]. 2021. http:// www.who.int/mediacentre/factsheets/fs297/en/

[3] Falah R, Golzari M, Dastani M, Zahir Aldin A, Musavi M, Akbari S. [The effectiveness of spirituality group training on increasing hopefulness and mental health in women with breast cancer (Persian)]. J Thought Behav Clin Psychol. 2011; 5(19):69-80. https://www.sid.ir/fa/journal/ ViewPaper.aspx?id=135458

[4] González-Fernández S, Fernández-Rodríguez C, Mota-Alonso MJ, García-Teijido P, Pedrosa I, Pérez-Álvarez M. Emotional state and psychological flexibility in breast cancer survivors. European J of Oncol Nurs. 2017; 30:75-83. [DOI: 10.1016/j.ejon.2017.08.006]

[5] Aaronson NK, Mattioli V, Minton O, Weis J, Johansen C, Dalton SO, et al. Beyond treatment-psychosocial and behavioural issues in cancer survivorship research and practice. EJC Suppl. 2014; 12(1):54-64. [DOI: 10.1016/j.ejcsup.2014.03.005]

[6] Donovan-Kicken E, Caughlin JP. Breast cancer patients' topic avoidance and psychological distress: The mediating role of coping. J Health Psychol. 2011; 16(4):596-606. [DOI: 10.1177/1359105310383605]

[7] Perpina C, Segura M, Sánchez-Reales S. Cognitive flexibility and decision-making in eating disorders and obesity. Eat Weight Disord. 2016; 22(3):435-44. [DOI: 10.1007/s40519-016-0331-3]

[8] Asnaashari K, Ghasemi SA. Comprehensive guide for patients with breast cancer: Diagnosis, treatment, nutrition, social and psychological problems. Tehran: Tabib; 2014.

[9] Shapiro SL, Carlson LE, Astin JA, Freedman B. Mechanisms of mindfulness. J Clin Psychol. 2006; 62(3):373-86. [DOI: 10.1002/jclp.20237]

[10] Cormio C, Romito F, Viscanti G, Turaccio M, Lorusso V, Mattioli V. Psychological well-being and posttraumatic growth in caregivers of cancer patients. Front Psychol. 2014; 5:1342. [DOI: 10.3389/fpsyg.2014.01342]

[11] Sharma A, Zhang J. Predictors of post traumatic growth among breast cancer patients in Nepal. Asian Pac J Health Sci. 2017; 4(2):918. [DOI: 10.21276/apjhs.2017.4.2.3]

[12] Calhoun LG, Tedeschi RG. Handbook of posttraumatic growth: Research and practice. England: Routledge; 2014. https://www.routledgehandbooks.com/doi/10.4324/9781315805597

[13] Janusek LW, Tell D, Mathews HL. Mindfulness based stress reduction provides psychological benefit and restores immune function of women newly diagnosed with breast cancer: A randomized trial with active control. Brain Behav Immun. 2019; 80:358-73. [DOI: 10.1016/j. bbi.2019.04.012]

[14] Katz LS. Holographic reprocessing: A cognitive-experiential psychotherapy for the treatment of trauma. $1^{\text {th }} \mathrm{ed}$. England: Routledge; 2015. https://www.routledge.com/Holographic-Reprocessing-A-CognitiveExperiential-Psychotherapy-for-the/author/p/book/9781138872769

[15] Katz, LS. Holographic reprocessing couple therapy with military couples. In: Pitta PJ, Datchi CC, editors. Integrative couple and family therapies: Treatment models for complex clinical issues. Washington: American Psychological Association; 2019. [DOI:10.1037/0000151007]
[16] Danhauer SC, Crawford SL, Farmer DF, Avis NE. A longitudinal investigation of coping strategies and quality of life among younger women with breast cancer. J Behav Med. 2009; 32(4):371-9. [DOI: 10.1007/s10865-009-9211-x]

[17] Narimani M, Kazemi N, Basharpoor S. [The effectiveness of holographic reprocessing therapy on traumatic memories and posttraumatic cognitions in women asking for divorce (Persian)]. J Couns Res. 2017 16(61):35-59. http://irancounseling.ir/journal/article-1-158-fa.htm

[18] Rezapour MY, Zakeri M. [The effectiveness of holographic re-treatment on the meaning of life, fear of negative evaluation and rumination of depressed women (Persian)]. Couns Cult Psychother. 2019; 10(39):4970. [DOI: 10.22054/QCCPC.2019.40602.2095]

[19] Salehi MN, Beshlideh K. [The effectiveness of holographic reprocessing therapy on cognitive flexibility, affective control and social adjustment in depressive patients with attempted suicide in llam city (Persian)]. Couns Cult Psychother. 2020; 11(43):183-216. [DOI: 10.22054/ QCCPC.2020.50615.2338]

[20] Katz LS, Cojucar G, Hoff RA, Lindl C, Huffman C, Drew T. Longitudina outcomes of women veterans enrolled in the Renew sexual trauma treatment program. J Contemp Psychother. 2015; 45:143-50. [DOI: 10.1007/s10879-014-9289-5]

[21] Mehrparvar S, Hajloo N, Abolghasemi A. [The effectiveness of holographic reprocessing therapy on mental adjustment to cancer in women with cancer (Persian)]. URMIA MED J. 2016; 28(5):343-52. http:// eprints.umsu.ac.ir/id/eprint/2905

[22] Delavar A. Research method in psychology and educational sciences. Tehran: Virayesh; 2019.

[23] Dennis JP, Vander Wal JS. The cognitive flexibility inventory: Instrument development and estimates of reliability and validity. Cognit Ther Res. 2010; 34:241-53. [DOI: 10.1007/s10608-009-9276-4]

[24] Shareh H, Farmani A, Soltani E. Investigating the reliability and valid ity of the Cognitive Flexibility Inventory (CFI-I) among Iranian university students. Pract Clin Psychol. 2014; 2(1):43-50. http://jpcp.uswr.ac.ir/ article-1-163-en.html

[25] Tedeschi RG, Calhoun LG. The posttraumatic growth inventory: Measuring the positive legacy of trauma. J Trauma Stress. 1996; 9(3):455-71. [DOI: 10.1007/BF02103658]

[26] Heidarzadeh M, Rassouli M, Mohammadi Shahbolaghi F, Alavi Majd H Mirzaeei HR, Tahmasebi M. [Validation of the Persian version of the Posttraumatic Growth Inventory in patients with cancer (Persian)]. Payesh. 2015; 14(4):467-73. [http://payeshjournal.ir/article-1-228-fa.html]

[27] Peak NJ. Book review: Holographic reprocessing as a treatment for military sexual trauma. J Contemp Psychother. 2015; 45:191-2. [DOI: 10.1007/s10879-015-9301-8]

[28] Joseph S, Murphy D, Regel S. An affective-cognitive processing mode of post-traumatic growth. Clin Psychol Psychother. 2012; 19(4):316-25. [DOI: 10.1002/cpp.1798]

[29] Mosher CE, Secinti E, Li R, Hirsh AT, Bricker J, Miller KD, et al. Acceptance and commitment therapy for symptom interference in metastatic breast cancer patients: A pilot randomized trial. Support Care Cancer. 2018; 26:1993-2004. [DOI: 10.1007/s00520-018-4045-0]

[30] Firouzi R, Tizdast T, Khalatbari J, Ghorban Shiroudi SH. [Relationship between stress coping strategies and difficulties in emotion regulation mediated by marital life quality in married women with breast cancer (Persian)]. J Arak Uni Med Sci. 2020; 23(1):34-47. [DOI: 10.32598/ JAMS.23.1.6005.1] 
This Page Intentionally Left Blank 